Methods 22 smokers (sm), 10 ex-smokers (ex-sm) and 21 never smokers (ns) with asthma performed spirometry, induced sputum and completed asthma control questionnaires pre and post an oral corticosteroid trial. Sputum fluid cytokines were quantified using a 25-plex bead system (Invitrogen, Paisley). Factor analysis was performed (SPSS V.17) using principal component analysis and Varimax rotation. Factors were identified according to; visual inspection of the scree plot, eigenvalues $>1.1$, minimum of three cytokines loading $>0.4$. Sequential removal of cytokines was performed in stages according to; moderate to strong $(>0.4)$ loadings, followed by requirement for 'strong' loading $(>0.6)$ to only one factor then removal of cytokines that reduced the reliability of the data set. In a final step cytokines with the lowest loadings were removed if a factor had $>3$.

Results The subjects were well matched except for higher asthma control questionnaires scores and inhaled corticosteroid dose in sm. $\mathrm{Sm}$ failed to demonstrate a lung function response to oral corticosteroids in contrast to nsm. No sputum cell differential differences were evident between smokers and non-smokers with asthma. A number of pre-steroid sputum cytokines were elevated in sm compared to nsm. The greatest difference present for interleukin 6 $(\mathrm{sm} 34.4 \mathrm{pg} / \mathrm{ml}$ (IOR 14.1, 72.4), $\mathrm{nsm} 8.1 \mathrm{pg} / \mathrm{ml}$ (4.4, 11.1), $\mathrm{p}<0.001)$. Factor analysis of the pre-steroid cytokines demonstrated that three factors explained $90 \%$ of the variance in the data. Sequential processing revealed three cytokines per factor (Abstract S122 table 1).

Discussion Sputum cytokine profiling of subjects with asthma with differing smoking histories reveals distinct groupings when examined by exploratory factor analysis providing insight into airway inflammation in asthma and the impact of smoking. Larger cohorts of patients with asthma should be examined to confirm these preliminary findings.

\section{S123 THE EFFECT OF RHINOVIRUS INFECTION ON COUGH RECEPTORS ON HUMAN SENSORY NERVE AND HUMAN PRIMARY BRONCHIAL EPITHELIAL CELLS}

doi:10.1136/thoraxjnl-2011-201054b.123

H Abdullah, S L Cosby, L Heaney, L McGarvey. Centre for Infection and Immunity, Queen's University Belfast, Belfast, UK

Human rhinovirus (HRV), a member of the picornaviridae family is a single stranded RNA virus. Rhinovirus infection in non-asthmatics rarely causes serious problems. However in asthmatic subjects HRV contributes to more than $60 \%$ of asthma exacerbations where the cough reflex is hyper-reactive and provokes severe coughing and wheezing.

Rational Members of a novel transient receptor potential (TRP) channels, the TRP vanilloid 1 (TRPV1), ankyrin like protein with transmembrane like domain 1 (TRPA1) and TRP melastatin 8 (TRPM8) have been shown to be involved in physiological and pathological aspects of cough. Subsequently understanding the interaction between HRV and 'cough receptors' is crucial as it may indicate potential therapeutic targets and strategies to block these interactions. In this study we investigated the effect of HRV infection on the cough reflex by determining the expression of receptors implicated in the cough process. We hypothesised that HRV may directly and/or indirectly interact with these receptors on sensory nerves and epithelial cells in the airways to provoke cough reflex.

Methods Human primary bronchial epithelial cells (PBEC) were obtained following informed consent and the human neuroblastoma (IMR-32) cell line was used to represent the fundamental cell type which controls the reflex to cough. The IMR-32 cells undergo differentiation (dIMR-32) to acquire characteristics of peripheral nerve cells with positive neuronal markers. The expression of 'cough receptors' at the protein level in both cell types were detected by fluorescent staining using confocal microscopy and flow cytometry analysis. Receptors mRNA levels were measured by quantitative real-time PCR at different time points post-infection with HRV or treatment with UV-inactivated virus or supernatant.

Results Both cell types dIMR-32 and PBEC were susceptible to HRV infection and showed positive staining for TRPA1, TRPV1 and TRPM8. Up-regulation of the 'cough receptors' mRNA occurred at low multiplicity of infection moreover, higher level of 'cough receptors' expression was detected in PBEC isolated from subjects with lung disease compared to healthy volunteers.

Conclusions These results suggest that virus may both induce cough and interfere with cough related airway clearance depending on the level of infection at different time points.

\section{S124 RAGE IS EXPRESSED BY HUMAN AIRWAY SMOOTH MUSCLE CELLS AND EXPRESSION IS INCREASED IN ASTHMA}

doi:10.1136/thoraxjnl-2011-201054b.124

L Di Candia, E Gomez, J Challiss, C E Brightling, R Saunders. University of Leicester, Leicester, UK

Background Airway smooth muscle (ASM) dysfunction contributes to the airway hyper-responsiveness and airflow obstruction observed in asthma. The receptor for advanced glycosylation end products (RAGE) is a pattern recognition receptor activated by endogenous danger signals, including high mobility group box 1 (HMGB1). RAGE and HMGB1 are implicated in many pathophysiological states, such as diabetes and Alzheimer's disease. RAGE is abundantly expressed in healthy adult lung, where it might serve a homeostatic function, for example, in alveolar type I epithelial cells; however, RAGE expression/function in other airway cell-types is poorly characterised. We hypothesised that changes in RAGE and HMGB1 expression may contribute to ASM dysfunction in asthma. Therefore, RAGE/HMGB1 expression was investigated in human primary ASM cells.

Methods ASM was microdissected from bronchial biopsies and large airway specimens obtained at lung resection surgery. Ex-vivo ASM cells were characterised for a-smooth muscle actin and used between passages 2-5. Cells were serum deprived in ITS medium for 72-h prior to experimentation. RAGE and HMGB1 mRNA expression was measured using RT-PCR and qRT-PCR, and protein expression by western blotting, immunofluorescence and flow cytometry.

Results ASM cells were shown to express the full-length and three soluble RAGE transcripts by RT-PCR $(n=5)$, and an HMGB1 transcript by qRT-PCR $(n=6)$. By flow cytometry membrane-localised RAGE expression was shown to be significantly increased in ASM cells isolated from asthmatics $(18.0 \pm 5.4 \%$ of the ASM population expressed RAGE, $\mathrm{n}=6)$ vs non-asthmatics $(8.6 \pm 6.5 \%, \mathrm{n}=7$; $\left.{ }^{*} \mathrm{p}<0.05\right)$, and a trend towards decreased HMGB1 expression in asthmatics $(18.8 \pm 10.5 \%, \mathrm{n}=6)$ vs non-asthmatics $(35.7 \pm 19.1 \%$, $\mathrm{n}=6$ ) was observed; however, these values were not significantly different. RAGE and HMGB1 expression were confirmed by immunofluorescence and western blotting.

Conclusion Human ASM cells express RAGE and its ligand HMGB1 at the mRNA and protein levels. Membrane-localised RAGE protein expression is significantly increased and there is a trend towards a decrease in HMGB1 protein expression in ASM isolated from asthmatic vs non-asthmatic subjects. The contribution of these changes to ASM dysfunction in asthma requires further investigation. 